\title{
Chitosan: A new tool for managing Placenta Accreta Spectrum Disorders with uncontrolled hemorrhage
}

\author{
Najeh Hcini ${ }^{1 *}$, Wahab Abdallah ${ }^{1}$, Meredith Mathieu ${ }^{1}$, Fatma Maamri $^{1}$, Véronique Lambert ${ }^{1}$, Léo Pomar $^{2}$, Olivier Picone ${ }^{3}$ and Gabriel Carles $^{1}$ \\ ${ }^{1}$ Department of Obstetrics and Gynaecology, West French Guiana Hospital Center, French Guiana. CIC Inserm 1424 et DFR santé université Guyane \\ ${ }^{2}$ Materno-Fetal and Obstetrics Research Unit, Department "Woman-Mother-Child”, Lausanne University Hospital, Lausanne, Switzerland. \\ ${ }^{3}$ Assistance Publique-Hôpitaux de Paris, Hôpital Louis Mourier, Service de Gynécologie-Obstétrique, Colombes, France, Université de Paris; Inserm IAME-U1137, \\ Paris, France; Groupe de Recherche sur les Infections pendant la Grossesse (GRIG),Vélizy, France
}

\begin{abstract}
Purpose: Placenta accreta spectrum disorder (PASD) is a rare but life-threatening complication of pregnancy. We aimed to describe the use of Chitosan impregnated gauze when managing PASD complicated by uncontrolled bleeding.

Methods: We used a descriptive retrospective study including all patients diagnosed with a PASD treated with Chitosan in the Department of Obstetrics and Gynaecology, West French Guiana Hospital Center, French Guiana, during a period of 5 years (January 1, 2015 to December 31, 2019).

Results: We listed 8 cases of PASD managed with Chitosan: 2 active bleeding in cesarean scar pregnancy in the first trimester, 2 cases of PASD in the second trimester, and 4 cases in the third trimester with average age of 33.6 years. Chitosan-covered gauze was used in $4 / 8$ cases of conservative surgery, $3 / 8$ of cases before hysterectomy and one case of pelvic packing after hysterectomy. Bleeding was immediately controlled in $7 / 8$ of patients but recurred in 3 of them after removing the gauze. Chitosan successfully treated bleeding of cesarean scar pregnancy (2/2); avoided 2/5 inevitable hysterectomy and stopped bleeding after hysterectomy with failure of hemostatic sutures (pelvic packing). No maternal or neonatal deaths were reported, despite high maternal morbidity in this group.

Conclusion: chitosan is an effective and safe treatment modality in management of life-threatening hemorrhage caused by abnormal placentation when conventional treatment had failed particularly in case of coagulopathy. It can be interesting to consider in isolated health centers, unexperienced teams, or /and unexpected cases.
\end{abstract}

\section{Introduction}

Placenta accreta spectrum disorders (PASD) refer to the range of pathologic adherence of the placenta leading to a lack of normal plane of cleavage between the placenta and the uterine wall. It is an increasing obstetrical emergency causing life-threatening hemorrhage in connexion with the increase of cesarean section delivery rate [1].

Complications of this entity results in maternal morbidity and mortality [2,3], including large volume blood loss requiring transfusion, coagulopathy, hysterectomy, urologic complications [4], and admission to intensive care unit. Late complications include septic complications, hospital readmission, and multiple surgeries [5]. However, despite the seriousness of this complication, few therapeutic means are available, especially in low-income countries. Furthermore, evidence is currently in favor that Cesarean scar pregnancy in the first trimester is the early expression of PASD in the second and third trimester of pregnancy [6].

We previously reported our experience in successfully using Chitosan, a potent hemostatic agent derived from chitin, to treat severe obstetric bleeding induced by uterine atony, multiple vaginal tears, and bleeding after hemostatic hysterectomy [7]. Since 2015, we have been regularly continuing to use Chitosan to manage gynecological or obstetrical hemorrhage.

In this study, we aimed to describe the use of chitosan to manage placenta accreta spectrum with uncontrolled hemorrhage and to evaluate its effect on maternal outcome.

\section{Materials and methods}

We conducted descriptive retrospective study in the Department of Obstetrics and Gynaecology, Franck Joly Hospital, French Guiana during the period from January 1, 2015 to December 31, 2019.

\section{Inclusion criteria}

We included all cases of PASD, or cesarean scar pregnancy treated with Chitosan-coated Gauze, fabric-dressing Coated with Celox haemostatic granules, regardless of term of pregnancy or route of administration (transvaginal or laparotomy).

In the second and third trimester, PASD was confirmed by either histopathological diagnosis of placental invasion in hysterectomy specimens, clinical assessment of abnormal adherence of the placenta, or evidence of gross placental invasion at the time of surgery. FIGO classification for the clinical diagnosis of placenta accreta spectrum disorders was used to describe each clinical case [8].

${ }^{\star}$ Correspondence to: Dr Najeh Hcini, M.D, MSc, PhD student, Department of Obstetrics and Gynaecology, West French Guiana Hospital Center, SaintLaurent-du-Maroni 97320, French Guiana, France, E-mail: hcininajeh@gmail. com

Key words: cesarean scar pregnancy, chitosan, placenta accreta spectrum disorder, postpartum hemorrhage, utero-vaginal packing

Received: April 18, 2021; Accepted: May 03, 2021; Published: May 07, 2021 
In the first trimester, we used transvaginal US imaging by referent sonographer to establish the diagnosis of pregnancy implanted in Cesarean section scar.

\section{Exclusion criteria}

We excluded bleeding treated with chitosan other than that due to PASD. Placenta previa without proof of accreta has not been considered in this study. PASD not managed with Chitosan were excluded.

\section{Study protocol}

In our delivery units, we used an active management of the third stage of labor, which consists of the prophylactic administration of an uterotonic agent prior to placental separation, early cord clamping, and uterine massage. Blood loss in the third stage of labor was estimated using a collector bag.

Postpartum hemorrhage (PPH) is defined as blood loss of at least $500 \mathrm{~mL}$ after vaginal or $1000 \mathrm{~mL}$ following cesarean delivery within 24 hours postpartum.

Escalation of measures follows a stepwise approach based on guidelines for clinical practice from the French College of Gynaecologists and Obstetricians (CNGOF) in collaboration with the French Society of Anesthesiology and Intensive Care (SFAR) [9]. The protocol included uterotonic treatment first (intravenous oxytocin, Sulproston). Dependent on the persistence of bleeding, biological (hemoglobin levels, hemostatic status) and clinical evaluation, other treatments have been added including tranexamic acid, fibrinogen, fresh frozen plasma and blood cell transfusion. In French Guyana department we do not have interventional radiology unit for uterine artery embolization. Thus, we perform hemostatic surgery after failure of Bakri balloon to control active bleeding.

In the first trimester with diagnosis of cesarean scar pregnancy was most often treated by local Methotrexate injection and curettage was only performed in case of severe vaginal bleeding.

In our practice, chitosan was not proposed as a first-line treatment but rather a final rescue solution in cases of life-threatening hemorrhage. When bleeding persisted, chitosan was introduced as a last treatment after failure of at least two other medical conventional treatments to control bleeding. Chitosan-coated gauze (Celox gauze, Fabric Dressing Coated with Celox Haemostatic Granules, Medtrade Products Ltd., Crewe, CW1 6GL, UK) was $3 \mathrm{~m}$ long, $7.6 \mathrm{~cm}$ wide and has a mass of 40 grams. Dependent on the size of uterine cavity and the term of pregnancy, a piece or the complete gauze was used.

An experienced senior obstetrician initially managed all cases. Collegial decision including two obstetrics and anesthesiologist is always required before the use of chitosan and/or the decision to perform a hysterectomy. In case of conservative treatment, the adherent part of the placenta was left in situ after delivery and uterus was closed.

In cases of vaginal or cesarean deliveries, the intrauterine packing was inserted transvaginally, up to the fundus, with ultrasound guidance and one end of the gauze was left into the vagina.

Chitosan-coated gauze was associated with Bakri balloon tamponade, after failure of the intrauterine balloon tamponade to control bleeding. In this case, first the chitosan-covered gauze was inserted, then the balloon replaced in the cavity, finally the vagina was packed by a second gauze for compression of cervix to avoid displacement of the balloon. Pain-reducing medications were administered as needed.
Intraoperatively, in cases of uncontrolled hemorrhage following hysterectomy and after failure of hemostatic sutures with intensive correction of coagulopathy, surgical packing using chitosan-coated Gauze was applied and left in place.

Chitosan-covered gauze was usually removed, in operative room, after 24-36 hours by simply pulling the end left in the vagina and observed for 2 hours after. They were given prophylactic antibiotic during this period. All patients were monitored until their six-week post-partum exam and given prophylactic anticoagulation in postpartum period.

\section{Data collection}

Information was collected on the characteristics of the mother, pregnancy, labor, mode of delivery, and postpartum period and the data entered on an Excel spreadsheet.

We collected information about post-partum or post post-abortion period (such as infection, pain, readmission, secondary surgery, and hospital stay) or any side effect of the treatment. The protocol was in accordance with the establishment's ethical charter.

\section{Results}

In all, 8 cases of PASD managed with Chitosan were retrospectively collected. During this period, 14205 live births have been registered. The mean maternal age was 33.6 years (range, 27-38 years). In this population, $14 \%$ of patients had at least one history of cesarean section and $2.64 \%$ had three C-section and more. Patient characteristics, PASD description, treatment and maternal outcome parameters are depicted in Table 1 .

In the first trimester, two patients were diagnosed with cesarean scar pregnancy at 8 weeks +2 days of gestation and 12 weeks of gestation. They were treated by methotrexate with monitoring of plasmatic hCG. Despite good biological evolution, they presented active bleeding. Under general anesthesia, suction curettage under ultrasound guidance was performed Treatment with uterotonic and tranexamic acid had failed to stop active hemorrhage estimated more than one liter. We used small packing with a piece of chitosan-covered gauze introduced in the uterine cavity at the level of the implantation site under ultrasound guidance. One from the two-patient had laceration of the cervix and needed suture with second gauze left in the vagina to pack the cervix. The bleeding stopped and gauze was removed 1 day after without complication. One of the two patients required transfusion of two red blood cells.

In the second trimester, we reported two patients with history of cesarean section. The first was suspected of PASD after her first ultrasound examination at 14 weeks +1 days of gestation. Pregnancy ended with spontaneous abortion with active bleeding complicated with hemodynamic instability and hemoglobin dropped to $4 \mathrm{~g} / 100 \mathrm{ml}$. Uterine aspiration under ultrasound guidance was performed and complicated by intraoperative hemorrhage. The patient displayed extensive bleeding (4-liter), hemodynamic instability and received massive transfusion. After eliminated perforation of the uterus and failure of uterotonic, tranexamic acid and Foley balloon tamponade to control bleeding, we decided to make intra uterine packing by chitosancovered gauze. The bleeding stopped immediately. Twenty-four later, after transfusion and correction of coagulopathy, total hysterectomy was done with good evolution.

The second parturient, living in an isolated commune, bi scarred uterus; was referred to our emergency for minimal bleeding. Fetal death 
Table 1. Baseline characteristics and pregnancy outcomes for patients with abnormal placentation treated with Chitosan-covered gauze

PASD, Placenta Accreta Spectrum Disorder; DIC, Disseminated intravascular coagulation; GA, gestational age; d, days; wk, week; y, years; G, gravida; P, para; CS, caesarean section. FIGO classification for the clinical diagnosis of placenta accreta spectrum disorders was used to describe each clinical case [7].

\begin{tabular}{|c|c|c|c|c|c|c|c|}
\hline $\begin{array}{l}\text { Case } \\
\text { (NO.) }\end{array}$ & $\begin{array}{l}\text { Age } \\
(\mathbf{y})\end{array}$ & $\begin{array}{l}\text { Gravidity and } \\
\text { Parity }\end{array}$ & $\begin{array}{l}\text { Prior } \\
\text { CS }\end{array}$ & $\underset{(w k+d)}{\text { GA }}$ & PASD description & Treatment, complications & Mode of administration \\
\hline 1 & 32 & G4P3 & 3 & $8+2$ & Cesarean scar pregnancy & $\begin{array}{c}\text { Methotrexate, active bleeding, Suction curettage, Uterotonic, } \\
\text { Tranexamic acid }\end{array}$ & Intra uterine packing \\
\hline 2 & 34 & G5P4 & 2 & 12 & Cesarean scar pregnancy & $\begin{array}{l}\text { Methotrexate, Active bleeding } \\
\text { Suction curettage, transfusion }\end{array}$ & Intra uterine packing \\
\hline 3 & 27 & G8P6 & 2 & $14+1$ & $\begin{array}{l}\text { Grade 2: Abnormally invasive } \\
\text { placenta (Increta) }\end{array}$ & $\begin{array}{c}\text { Active bleeding, Uterine aspiration, Foley balloon tamponade, } \\
\text { Hemodynamic instability, Coagulopathy } \\
\text { transfusion }\end{array}$ & $\begin{array}{l}\text { Intra uterine packing to stop } \\
\text { bleeding, Hysterectomy after } \\
\text { removing Chitosan-covered gauze } \\
\text { ( } 24 \text { hours) }\end{array}$ \\
\hline 4 & 33 & G9P6 & 1 & $21+2$ & $\begin{array}{l}\text { Grade 2: Abnormally invasive } \\
\text { placenta (Increta) }\end{array}$ & $\begin{array}{l}\text { Active bleeding, } \\
\text { Hemodynamic instability, } \\
\text { Coagulopathy, } \\
\text { transfusion }\end{array}$ & $\begin{array}{l}\text { Intra uterine packing, } \\
\text { Hysterectomy after removing } \\
\text { Chitosan-covered gauze ( } 48 \\
\text { hours) }\end{array}$ \\
\hline 5 & 35 & G10P8 & 4 & $37+2$ & $\begin{array}{l}\text { Grade 3a: Abnormally invasive } \\
\text { placenta (Percreta) }\end{array}$ & $\begin{array}{l}\text { Massive transfusion, } \\
\text { Coagulopathy, } \\
\text { Hemodynamic instability, } \\
\text { Failure of hemostatic sutures }\end{array}$ & $\begin{array}{l}\text { Hysterectomy, } \\
\text { Packing of the pelvic cavity, } \\
\text { Antibiotics }\end{array}$ \\
\hline 6 & 38 & G5P4 & 2 & 37 & $\begin{array}{l}\text { Grade 2: Abnormally invasive } \\
\text { placenta (Increta) }\end{array}$ & $\begin{array}{l}\text { Massive transfusion, Uterine artery ligation, hemodynamic } \\
\text { instability, DIC, Factor VIIa recombinant administration }\end{array}$ & $\begin{array}{l}\text { Intra uterine packing, } \\
\text { Hysterectomy }\end{array}$ \\
\hline 7 & 32 & G6P5 & 4 & $37+2$ & $\begin{array}{l}\text { Grade 2: Abnormally invasive } \\
\text { placenta (Increta) }\end{array}$ & $\begin{array}{c}\text { Massive transfusion, Coagulopathy, Failure of Sulproston and } \\
\text { Balloon tamponade, Uterine artery ligation }\end{array}$ & $\begin{array}{l}\text { Chitosan-covered gauze combined } \\
\text { with Intrauterine balloon } \\
\text { tamponade, vaginal and cervical } \\
\text { packing. }\end{array}$ \\
\hline 8 & 38 & G8P8 & 5 & 38 & $\begin{array}{l}\text { Grade 2: Abnormally invasive } \\
\text { placenta (Increta) }\end{array}$ & $\begin{array}{c}\text { Failure of Sulproston, Uterine artery ligation, Massive } \\
\text { transfusion }\end{array}$ & Intra uterine packing \\
\hline
\end{tabular}

in utero at 21 weeks +2 days of gestation was diagnosed and placenta was described as anterior far from the cervix with oligohydramnios. The patient was given Mifepritone and induced, 48 hours after, with misoprostol to make normal delivery. Because of Failure of cervical maturation and appearance of severe infection with sepsis, laparotomy has been decided and was done in emergency. Intraoperative unexpected placenta accreta was discovered and managed conservatory. Because of infection and severe bleeding, rapid disturbed hemostasis happened, the patient's hemodynamic status worsened. The patient had lost more than 2-liter requiring massive blood transfusion. We used intra uterine packing with Chitosan-coated gauze. The treatment was effective, and bleeding completely stopped. Secondarily when removing the Celox Gauze, 48 hours later, patient underwent hysterectomy without complication for recurrent bleeding.

In the third trimester, we listed 4 cases of PASD managed by chitosan-covered gauze when bleeding persisted after delivery. Three from them have been diagnosed in antenatal ultrasound. Among these patients, chitosan-covered gauze was used successfully in two cases of conservative surgery after all other treatment have failed. Consequently, it avoided laparotomy/hysterectomy that, in our opinion, had become almost inevitable.

We report specially the case of a 32-year old patient, bi-scarred uterus with suspicion of placenta accreta and desire to conserve the uterus. We opted for conservative treatment and the adherent part of placenta was left in the uterus. After failure of all treatment to stop bleeding including uterine artery ligation, administration of uterotonics, and balloon tamponade, hysterectomy (HE) seemed inevitable otherwise. The patient required transfusion of three units of packed red blood cells and two of fresh frozen plasma after bleeding more than 2 liter. The patient developed coagulopathy. Given the persistent bleeding, we associated chitosan-covered gauze with intrauterine balloon tamponade and vaginal packing. The bleeding stopped and all packing was removed 24 hours after with good evolution.
In one case, pelvic packing using chitosan-covered gauze was performed. Hemostatic hysterectomy for placenta accreta was performed and complicated with life-threatening hemorrhage, disseminated intravascular coagulation, and massive transfusions. We estimated that she had lost more than 5 liters of blood and her hemoglobin concentration fell to $4 \mathrm{~g} / 100 \mathrm{~mL}$. Surgical hemostasis was insufficient to control bleeding in the surgical site. Packing of the pelvic cavity using Chitosan-coated Gauze was successful and bleeding stopped completely. The gauze was removed, without difficulty, during a surgical intervention two days later. Surgeon founded no bleeding, and no complication was reported in the post-partum period.

During the follow-up period of 6 weeks, there was no readmission, endometritis, in any of these patients. Patients with conservative treatment did not report changes of the menstrual period or abnormal bleeding. Two patients were pregnant after treatment without recurrence. There was no maternal death during this period.

\section{Discussion}

PASD are no longer a rare situation in obstetrics and its incidence have been increasing in recent years [1]. Most of these entities are found in women presenting a previous history of caesarean section and a placenta praevia [10]. As well as serious maternal morbidity increases progressively with increasing number of cesarean deliveries [11], the risk of placenta accreta increase with the number of uterine scars in case of placenta previa [12]. PASD is associated with severe postpartum hemorrhage resulting in a mortality rate of approximately $7 \%[13,14]$. Thanks to study in naturel history of patient with Cesarean scar pregnancy who desire to continue the pregnancy, evidence is currently in favor that this entity in the first trimester is the early expression of PASD in the second and third trimester of pregnancy. They are no longer two separate pathologies, but rather the consequences of a single abnormality $[6,15,16]$. 
Chitosan is a hydrophilic biopolymer obtained by the chemical deacetylation of chitin, the principal component of crustacean shells. Chitosan exists in three forms: granules, gauze, and nasal plugs. It has been used for several years by military doctors to stop the bleeding of combat wounds $[17,18,19]$. It received approval for medical use in the United States in 2003. Other uses are being developed, including traumatic injuries in civilians [20], hemostasis during surgery [21], the prevention of recalcitrant epistaxis [22] and in cases of severe obstetric hemorrhage $[23,6]$.

Interestingly, this is the first study that has specifically addressed the experience of using Chitosan-covered gauze in the management of PASD. We founded that Chitosan is efficient, in combination with multiple adjuvant treatment modalities, to control massive hemorrhage secondary to PASD. It is also the first report of successful use of "uterine sandwich" approach described by Seidel et al. [24] for uterine atony in the conservative treatment of placenta accreta. This technique is interesting to consider in case of placenta accreta and desired fertility preservation.

It is well documented that placenta accreta spectrum is better managed by interdisciplinary teams in experienced centers [25], however it is not always possible in cases of some unexpected cases or in isolated centers. To improve the outcomes of this emergency, obstetric units and practitioners must have equipment in place to manage this emergency properly [26]. This maternal mortality and morbidity are sometimes secondary to the insufficiency of therapeutic means and/ or the lack of experience of the operator. Despite therapeutic progress, mortality and morbidity of PASD is still high worldwide [27,28]. Chitosan can be a solution to improve maternal outcomes of this highrisk obstetric entity, no longer rare in our practice. The most important factor with an approach using Chitosan that it does not require a learning curve or prior training $[6,28]$. In our experience, chitosan is not the miraculous solution to avoid hysterectomy in all case of PASD; it can be helpful in case the clinical situation is critic to stop bleeding in acute phase. Thus, chitosan can be a way to control bleeding before transferring the patient in adapted center or to achieve embolization of uterine artery.

Based on previous studies, chitosan-coated gauze is a safe and successful treatment modality when managing severe cases of postpartum hemorrhage [6,28]. Its effectiveness is probably explained by both compressing the mechanical compression of lower uterine segment, adhesion of the gauze and improved local hemostasis which is independent of the coagulation cascade. Compressing the lower uterine segment is important in preventing or stopping $\mathrm{PPH}$, particularly when the bleeding is due to placenta previa $[28,29]$. In this study, as well as other articles $[21,30]$, we reported no side effects and no specific treatment-associated morbidity during or after the procedure even in case of shellfish allergic subjects [31]. Concerning the economic aspect, its costs are one fifth those of a Bakri intrauterine balloon (50 euros/250 euros). Thus, Chitosan can be suitable for low resource countries and in the absence of embolization. The association of the two treatments can also be an ultimate solution before surgery especially when in case of cervical bleeding.

In our opinion, we support the introduction of Chitosan, among therapeutic arsenal already at our disposal, when dealing with bleeding caused by abnormal placentation, which are often difficult to treat surgically. The main limitation of this study is obviously the sample size. We need multicentric and comparative study to determine the exact place of Chitosan when managing PASD.

\section{Conclusion}

We conclude that Chitosan is a new effective therapeutic arsenal to treat uncontrolled hemorrhage secondary to abnormal placentation when conventional resources have failed. It can be an ultimate solution to stop bleeding, temporize to transfer the patient or to correct hemostasis. It is inexpensive, easy to apply and has no side effects. Chitosan is interesting to consider in the management of this high-risk condition especially in isolated health centers, unexperienced teams or /and unexpected cases.

\section{Authorship and contributorship}

All authors contributed to the manuscript.

Najeh Hcini: Data collection or management, Data analysis, Manuscript writing.

Wahab Abdallah, Meredith Mathieu, Fatma Maamri: Data collection, Data analysis.

Véronique Lambert, Léo Pomar, Olivier Picone: Manuscript writing/editing, Data analysis.

Gabriel Carles: reviewed the literature, Protocol/project development, Data analysis.

\section{Acknowledge}

none.

\section{Funding}

No external funding was used in this conduct of this study.

\section{Conflicts of interest/Competing interests}

The authors declare that there is no conflict of interest.

\section{References}

1. Wu S, Kocherginsky M, Hibbard JU (2005) Abnormal placentation: twenty-year analysis. Am J Obstet Gynecol 192: 1458-1461. [Crossref]

2. Usta IM, Hobeika EM, Musa AA, Gabriel GE, Nassar AH (2005) Placenta previaaccreta: risk factors and complications. Am J Obstet Gynecol 193: 1045-1049. [Crossref]

3. Shellhaas CS, Gilbert S, Landon MB, Varner MW, Leveno KJ, et al. (2009) The frequency and complication rates of hysterectomy accompanying cesarean delivery. Obstet Gynecol 114: 224-229. [Crossref]

4. Washecka R, Behling A (2002) Urologic complications of placenta percreta invading the urinary bladder: a case report and review of the literature. Hawaii Med J 61: 66 -69. [Crossref]

5. Morel O, Desfeux P, Fargeaudou Y, Malartic C, Rossignol M, et al. (2009) Uterine conservation despite severe sepsis in a case of placenta accreta first treated conservatively: 3-month delayed successful removal of the placenta. Fertil Steril 91: 1957.e5-9. [Crossref]

6. Zosmer N, Fuller J, Shaikh H, Johns J, Ross JA (2015) Natural history of early first-trimester pregnancies implanted in Cesarean scars. Ultrasound Obstet Gynecol 46: 367-375.

7. Carles G, Dabiri C, Mchirgui A, Saoudi EO, Hcini N, et al. (2017) Uses of chitosan for treating different forms of serious obstetrics hemorrhages. J Gynecol Obstet Hum Reprod 46: 693-695. [Crossref]

8. Jauniaux E, Ayres-de-Campos D, Langhoff-Roos J, Fox KA, Collins S, et al. (2019) FIGO classification for the clinical diagnosis of placenta accreta spectrum disorders. Int J Gynaecol Obstet 146: 20-24. [Crossref]

9. Sentilhes L, Vayssière C, Deneux-Tharaux C, Aya AG, Bayoumeuet F, et al. (2016) Postpartum hemorrhage: guidelines for clinical practice from the French College of Gynaecologists and Obstetricians (CNGOF): in collaboration with the French Society of Anesthesiology and Intensive Care (SFAR). Eur J Obstet Gynecol Reprod Biol 198: 12-21. [Crossref] 
10. Jauniaux E, Jurkovic D (2012) Placenta accreta: pathogenesis of a 20th century iatrogenic uterine disease. Placenta 33: 244-251. [Crossref]

11. Silver RM, Landon MB, Rouse DJ, Leveno KJ, Spong CY, et al. (2006) Maternal morbidity associated with multiple repeat cesarean deliveries. Obstet Gynecol 107: 1226-1232. [Crossref]

12. Clark SL, Koonings PP, Phelan JP (1985) Placenta previa/accreta and prior cesarean section. Obstet Gynecol 66: 89-92. [Crossref]

13. Kayem G, Davy C, Goffinet F, Thomas C, Clement D, et al. (2004) Conservative versus extirpative management in cases of placenta accreta. Obstet Gynecol 104: 531-536. [Crossref]

14. Haeri S, Dildy 3rd GA (2012) Maternal mortality from hemorrhage. Semin Perinatol 36: 48-55.

15. Cali G, Forlani F, Timor-Tritsch IE, Palacios-Jaraquemada J, Minneci G, et al. (2017) Natural history of Cesarean scar pregnancy on prenatal ultrasound: the crossover sign. Ultrasound Obstet Gynecol 50: 100-104.

16. Zosmer N, Fuller J, Shaikh H, Johns J, Ross JA (2015) Natural history of early first-trimester pregnancies implanted in Cesarean scars. Ultrasound Obstet Gynecol 46: 367-375.

17. Pozza M, Millner RWJ (2011) Celox (chitosan) for haemostasis in massive traumatic bleeding: experience in Afghanistan. Eur J Emerg Med 18: 31-33. [Crossref]

18. Zhang YJ, Gao B, Liu XW (2015) Topical and effective hemostatic medicines in the battlefield. Int J Clin Exp Med 8: 10-19. [Crossref]

19. whinney AC, Kirk SJ (2015) A systematic review of the use of tourniquets and topical haemostatic agents in conflicts in Afghanistan and Iraq. $J$ R Nav Med Serv 101: 147 154. [Crossref]

20. Hatamabadi HR, Asayesh Zarchi F, Kariman H, Arhami Dolatabadi A, Tabatabaey A et al. (2015) Celox-coated gauze for the treatment of civilian penetrating trauma: a randomized clinical trial. Trauma Mon 20: e23862. [Crossref]

21. Arul GS, Bowley DM, DiRusso S (2012) The use of Celox gauze as an adjunct to pelvic packing in otherwise uncontrollable pelvic haemorrhage secondary to penetrating trauma. J R Army Med Corps 158: 331-333. [Crossref]
22. Kourelis K, Shikani AH (2012) Effectiveness of chitosan-based packing in 35 patients with recalcitrant epistaxis in the context of coagulopathy. Clin Otolaryngol 37: 309313. [Crossref]

23. Schmid BC, Rezniczek GA, Rolf N, Saade G, Gebauer G, et al. (2013) Uterine packing with chitosan-covered gauze for control of postpartum hemorrhage. Am J Obstet Gynecol 209: 225e1-225e5. [Crossref]

24. Seidel V, Braun T, Weizsäcker K, Henrich W (2018) Application of chitosan-covered gauze in combination with intrauterine balloon tamponade for postpartum hemorrhage treatment - Case report of a novel "uterine sandwich" approach. Int J Surg Case Rep 48: 101-103. [Crossref]

25. Eller AG, Bennett MA, Sharshiner M, Masheter C, Soisson AP, et al. (2011) Maternal morbidity in cases of placenta accreta managed by a multidisciplinary care team compared with standard obstetric care. Obstet Gynecol 117: 331-337. [Crossref]

26. American College of Obstetricians and Gynecologists. Obstet Gynecol. 2006 Oct;108(4):1039-47. ACOG Practice Bulletin: Clinical Management Guidelines for Obstetrician-Gynecologists Number 76, October 2006: postpartum hemorrhage.

27. Jauniaux E, Grønbeck L, Bunce C, Langhoff-Roos J, Collins SL (2019) Epidemiology of placenta previa accreta: a systematic review and meta-analysis. BMJ Open 9: e031193. [Crossref]

28. Farquhar CM, Li Z, Lensen S, McLintock C, Pollock W, et al. (2017) Incidence, risk factors and perinatal outcomes for placenta accreta in Australia and New Zealand: a case-control study. BMJ Open 7: e017713.

29. Cho HY, Park YW, Kim YH, Jung I, Kwon JY (2015) Efficacy of intrauterine bakri balloon tamponade in cesarean section for placenta previa patients. PLoS One 10: e0134282. [Crossref]

30. Dueckelmann AM, Hinkson L, Nonnenmacher A, et al. (2019) Uterine packing with chitosan-covered gauze compared to balloon tamponade for managing postpartum hemorrhage. Eur J Obstet Gynecol Reprod Biol 240: 151-155. [Crossref]

31. Waibel KH, Haney B, Moore M, Whisman B, Gomez R (2011) Safety of chitosan bandages in shellfish allergic patients. Mil Med 176: 1153-1156. [Crossref]

Copyright: (C2021 Hcini N. This is an open-access article distributed under the terms of the Creative Commons Attribution License, which permits unrestricted use, distribution, and reproduction in any medium, provided the original author and source are credited. 